\title{
Xenon anesthesia: safe, protective and neurotoxic?
}

\author{
Gregory M.T. Hare MD
}

X

ENON is an inert noble gas found in trace amounts in the atmosphere, whose anesthetic properties have been known for over 30 years. ${ }^{1}$ The mechanism of xenon-induced anesthesia includes antagonism of neuronal $\mathrm{N}$-methylD-aspartate (NMDA) glutamate receptors. ${ }^{2}$ Early clinical trials of xenon anesthesia have been performed, with no clear evidence of adverse effects. ${ }^{3,4}$ The potential additional benefits of xenon anesthesia have been highlighted by more recent experimental studies which have demonstrated that xenon protects neonatal rat neurons from hypoxia/ischemia. In one study, xenon pre-conditioning reduced neurological injury and improved functional outcomes after hypoxia/ischemia in seven-day-old rat pups. ${ }^{5}$ In another study, xenon, in combination with hypothermia, more effectively protected against hypoxic/ischemic neuronal injury, when compared to hypothermia or xenon alone. ${ }^{6}$ Currently, the benefits and risks of xenon anesthesia continue to be studied in ongoing clinical and animal studies.

In this issue of the Journal, Cattano et al. ${ }^{7}$ utilize well established methods to assess the potential toxic effects of isoflurane and xenon on cerebral neurons in neonatal mice. Utilizing immunostaining techniques, they identify neurons which are "committing suicide" by labelling sections of mouse brain for the activated "suicide" enzyme, caspase- 3 . Activated caspase- 3 is a well recognized and specific marker of neurons which are undergoing programmed cell death, or neuroapoptosis. The results suggest that xenon can induce neuroapoptosis, as demonstrated by an increase in the number of activated caspase- 3 positive neurons in the cerebral cortex and basal ganglia (caudate/putamen) of seven-day-old mice. In a previous report, these investigators demonstrated that an increase in the number of activated caspase- 3 positive neurons was associated with impaired neurological function. ${ }^{8}$ In addition, isoflurane exposure results in a much larger increase in the number of activated caspase- 3 positive neurons, suggesting that isoflurane causes a higher degree of neuroapoptosis than xenon alone. Paradoxically, exposure to both xenon and isoflurane results in less neuroapoptosis than isoflurane alone, suggesting a potential neuroprotective role for xenon during isoflurane anesthesia.

These data provide a number of surprising insights: firstly, that a new and "safe" anesthetic has neurotoxic potential; secondly, that the established and "safe" anesthetic isoflurane has greater neurotoxic potential than xenon, and finally, that one anesthetic with neurotoxic potential (xenon) protects against neurotoxicity initiated by another anesthetic (isoflurane). The authors conclude that "xenon presents an enigma" in which the anesthetic gas is both neurotoxic and neuroprotective, under different experimental conditions.

It is of interest to clinicians that anesthetic agents with established safety profiles profoundly suppress neuronal function to induce the desired clinical effect of hypnosis. However, increasing evidence suggests that such suppression of neuronal activity may cause some neurons to activate internal suicide signals, leading to programmed cell death. ${ }^{9}$ This concern has recently been debated in the literature, after publication of an animal study which demonstrated an increase in neuroapoptosis and learning defects, in neonatal rats exposed to a combination of commonly administered anesthetic agents. ${ }^{8,10,11}$ Notably, Olney and colleagues have been at the forefront of understanding the potential of anesthetic drugs to cause neuronal injury. Their laboratory research has produced a number of important studies which demonstrate that anesthetic drugs which block NMDA glutamate receptors (nitrous oxide), or activate gamma aminobutyric acid (GABA) receptors (isoflurane) can induce neuronal cell death, particularly in neonatal rodents. The combination of an NMDA antagonist and a GABA agonist can result in a much higher level of neuroapoptosis. They showed that: exposure to NMDA antagonists

CAN J ANESTH $2008 / 55: 7 /$ pp 403-407

From the Departments of Anesthesia and Physiology, St. Michael's Hospital, Keenan Research Centre in the Li Ka Shing Knowledge Institute, University of Toronto, Toronto, Ontario, Canada.

Address correspondence to: Dr. Gregory Hare, Department of Anesthesia, St. Michael's Hospital, 30 Bond Street, Toronto, Ontario M5B 1W8, Canada. E-mail: hareg@smh.utoronto.on.ca

Competing interests: None declared. 
or isoflurane triggers neuroapoptosis in seven-dayold rats; $;{ }^{8,12,13}$ the combination of midazolam, nitrous oxide, and isoflurane, causes more pronounced neuroapoptosis and learning impairment in seven-day-old rats; ${ }^{8}$ and prolonged nitrous oxide exposure leads to neuronal death in adult rats. ${ }^{14}$ This landmark research has identified that neurons within the developing and adult rodent brain are susceptible to neuroapoptosis when exposed to common anesthetic agents. Why is this evidence emerging now, after years of utilizing these anesthetic agents? The explanation may lie in that fact that few published studies have addressed the question: Do anesthetic agents harm neurons? A search of the literature using the heading "anesthetics and neuron apoptosis" yielded 113 papers, while a search of "neuron apoptosis" produced 10,111 papers (Web of Science). Thus, few investigators are addressing this issue.

From the anesthesiologists' perspective, these studies are an obvious source of concern, as patients are safely anesthetized with these agents every day. Clinical experience has demonstrated that anesthesia is extremely safe. Recent reports demonstrate a significant reduction in the incidence of death and severe neurological injury that are attributable to anesthesia. ${ }^{15,16}$ This excellent safety record has been achieved through steady advances in clinic care, education, and monitoring. However, the occurrence of adverse neurological outcomes, including cognitive dysfunction ${ }^{17}$ and focal ischemia, ${ }^{18}$ continues to be a major source of postoperative morbidity. The potential factors contributing to these outcomes are numerous and may have more to do with the systemic response to the stress of surgery and variation in physiological parameters, than with the anesthetic agent utilized. Nevertheless, the evidence cited suggests that anesthetic agents could be responsible for causing selective neuronal loss in our patients. Identifying this phenomenon in humans would be very difficult, because the ability to detect such selective neuronal loss may be beyond the sensitivity of current medical imaging techniques. Furthermore, the functional deficit imparted by selective neuronal loss would be difficult to measure. In the pediatric population, this effect may be even more difficult to assess, as the full potential of the developing nervous system and individual may take years to be realized. However, if we don't ask the question, and test the hypothesis with sound experimental methodology, no answer will emerge.

The enthusiasm to develop new and safer anesthetics must be maintained to advance the field of anesthesia. Such enthusiasm is evident in the abstract of one experimental study which states that xenon has an "excellent safety profile" with "great promise as a neuroprotectant". ${ }^{6}$ Statements of this nature must be assessed with care. From the published literature, a search of "safety and anesthesia" yielded 352 articles in the past 20 years (Web of Science), while a search for "xenon and anesthesia" yielded 244 references. Collective searches for "isoflurane, propofol, midazolam, ketamine and nitrous oxide and anesthesia" yielded more than 20,000 studies. Yet, the potential for many of these agents to cause neuroapoptosis has been demonstrated in animal models. In addition, xenon has been shown to increase the incidence of nausea and vomiting, and to disrupt the balance of cerebral blood flow between white and grey matter in humans. ${ }^{19,20}$ Clearly, more studies are needed to determine the efficacy and safety of xenon anesthesia. Furthermore, the finding that established anesthetic agents can also induce neuroapoptosis, provides support for the concept of post-marketing surveillance in order to maintain ongoing vigilance for potential drug toxicity.

The efforts to use sophisticated molecular scientific approaches to develop safer and more effective anesthetics are exemplified in ongoing research by Orser, ${ }^{21}$ and others, who are defining specific subtypes of the GABA receptor which may provide important "targets" for future anesthetic drugs. Continued experimental and clinical studies, which assess the effect of anesthetics on neuronal health and injury, will provide a clearer understanding of the risks and benefits of anesthesia for our patients, and potentially lead to the development of novel and improved anesthetic agents.

\section{L'anesthésie au xénon : sécuritaire, protectrice et neurotoxique ?}

Le xénon est un gaz noble et inerte qu'on trouve en quantités minimes dans l'atmosphère et dont les propriétés anesthésiques sont connues depuis une trentaine d'années. ${ }^{1}$ Le mécanisme de l'anesthésie induite par le xénon comprend l'antagonisme des récepteurs du glutamate $\mathrm{N}$-méthyl-D-aspartate (NMDA) neuronaux. ${ }^{2}$ Lorsque les premières études cliniques portant sur l'anesthésie au xénon ont été réalisées, elles n'ont 
pas apporté de données probantes claires quant aux effets secondaires. ${ }^{3,4}$ Les bienfaits potentiels supplémentaires de l'anesthésie au xénon ont été soulignés par des études expérimentales plus récentes, lesquelles ont démontré que le xénon protège les neurones des rats nouveau-nés de l'hypoxie/ischémie. Dans une étude, un conditionnement préliminaire au xénon a permis de réduire les lésions neurologiques et a amélioré les résultats fonctionnels après une hypoxie/ ischémie chez des rongeurs nourrissons âgés de sept jours. ${ }^{5}$ Dans une autre étude, le xénon, en association avec l'hypothermie, a protégé plus efficacement contre une lésion neuronale hypoxique/ischémique, par rapport au xénon ou à l'hypothermie seul(e). ${ }^{6}$ À l'heure actuelle, les bienfaits et risques de l'anesthésie au xénon sont encore étudiés dans des études cliniques et animales en cours.

Dans ce numéro du Journal, Cattano et coll. ${ }^{7}$ se servent de méthodes bien établies pour évaluer les effets toxiques potentiels de l'isoflurane et du xénon sur les neurones cérébraux de rongeurs nouveau-nés. À l'aide de techniques d'immunocoloration, ils ont identifié les neurones "se suicidant» en marquant certaines sections du cerveau du rongeur pour l'enzyme activée du «suicide ", la caspase- 3 . La caspase-3 activée est un marqueur bien reconnu et spécifique des neurones subissant une mort cellulaire programmée, ou neuroapoptose. Ces résultats suggèrent que le xénon peut provoquer la neuroapoptose, tel que cela est démontré par une augmentation du nombre de neurones positifs à la caspase-3 activée dans le cortex cérébral et les noyaux gris centraux (noyau caudé/putamen) chez les rongeurs âgés de sept jours. Dans une étude précédente, ces auteurs ont montré qu'une augmentation du nombre de neurones positifs à la caspase- 3 activée était associée à une fonction neurologique perturbée. ${ }^{8}$ De plus, l'exposition à l'isoflurane engendre une augmentation bien plus importante du nombre de neurones positifs à la caspase-3 activée, ce qui laisse à penser que l'isoflurane provoque une neuroapoptose plus marquée que le xénon seul. Paradoxalement, une exposition au xénon et à l'isoflurane provoque moins de neuroapoptose que l'isoflurane seul, ce qui suggère que le xénon joue peut-être un rôle neuroprotecteur lors d'une anesthésie à l'isoflurane.

Ces résultats ouvrent plusieurs perspectives inattendues : tout d'abord, qu'un anesthésique nouveau et sécuritaire a un potentiel neurotoxique ; ensuite, que l'isoflurane, un anesthésique bien établi et "sécuritaire », possède un potentiel neurotoxique plus important que le xénon; enfin, qu'un anesthésique avec un potentiel neurotoxique, le xénon, protège contre la neurotoxicité provoquée par un autre anesthésique, l'isoflurane. Les auteurs concluent que «le xénon présente une énigme » dans laquelle le gaz anesthésique est à la fois neurotoxique et neuroprotecteur, selon les conditions expérimentales.

Pour le clinicien, il est intéressant de savoir que certains agents anesthésiques avec des profils d'innocuité établis suppriment profondément la fonction neuronale afin de provoquer l'hypnose, l'effet clinique désiré. Néanmoins, un nombre croissant de données suggèrent qu'une telle suppression de l'activité neuronale pourrait avoir pour résultat que certains neurones activent des signaux internes de suicide, provoquant une mort cellulaire programmée. ${ }^{9}$ Cette inquiétude a récemment été discutée dans la littérature, à la suite de la publication d'une étude animale démontrant une augmentation de la neuroapoptose et des difficultés d'apprentissage chez les rongeurs nourrissons exposés à une combinaison d'agents anesthésiques fréquemment utilisés. ${ }^{8,10,11}$ Olney et collègues en particulier sont à la tête des recherches qui ont pour objectif de mieux comprendre le potentiel des anesthésiques à causer des lésions neuronales. Leurs recherches de laboratoire ont donné lieu à plusieurs études importantes démontrant que les agents anesthésiques bloquant les récepteurs de glutamate NMDA (oxyde d'azote), ou activant les récepteurs d'acide gamma-aminobutyrique (GABA) (isoflurane) peuvent provoquer la mort cellulaire neuronale et ce, particulièrement chez les rongeurs nouveau-nés. L'association d'un antagoniste NMDA et d'un agoniste GABA provoque un taux beaucoup plus élevé de neuroapoptose. Les auteurs ont montré que : l'exposition à des antagonistes NMDA ou à de l'isoflurane déclenche la neuroapoptose chez les rongeurs de sept jours ; $;, 12,13$ l'association de midazolam, d'oxyde d'azote et d'isoflurane provoque une neuroapoptose plus prononcée et des difficultés d'apprentissage chez les rongeurs âgés de sept jours $;^{8}$ et une exposition prolongée à l'oxyde d'azote provoque une mort neuronale chez les rongeurs adultes. ${ }^{14}$ Ces recherches jalon ont permis de déterminer que les neurones dans le cerveau en développement et dans le cerveau adulte du rongeur peuvent être touchés par la neuroapoptose lorsqu'ils sont exposés à des agents anesthésiques courants. Comment se fait-il que ces données probantes voient le jour maintenant, alors que nous utilisons ces agents anesthésiques depuis des années? Une explication plausible est que peu d'études publiées ont tenté de répondre à la question suivante : les agents anesthésiques sont-ils nocifs pour les neurones? Dans une recherche de la littérature utilisant les termes " anesthetics and neuron apoptosis », 113 articles sont apparus, alors qu'avec le terme "neuron apoptosis », 10111 références sont revenues 
(Web of Science). Dès lors, il est clair que peu de chercheurs s'intéressent à cette question.

Du point de vue de l'anesthésiste, ces études constituent une source évidente d'inquiétude, étant donné que chaque jour des patients sont anesthésiés "de façon sécuritaire » avec ces agents. L'expérience clinique a démontré que l'anesthésie est extrêmement sécuritaire. Des comptes-rendus récents montrent une réduction considérable du nombre de décès et de lésions neurologiques graves attribuables à l'anesthésie. ${ }^{15,16}$ Ces excellentes conditions de sécurité sont le résultat de progrès continus dans les soins cliniques, la formation, et la surveillance des patients. Cependant, la survenue de devenirs neurologiques négatifs, notamment de dysfonctionnement cognitif ${ }^{17}$ et d'ischémie focale ${ }^{18}$ constitue encore une source importante de morbidité postopératoire. Les facteurs qui contribuent à ces résultats impliquent plusieurs facettes et pourraient être aussi bien liés à la réaction systémique au stress de la chirurgie et à la variation des paramètres physiologiques qu'à l'agent anesthésique utilisé. Néanmoins, les données probantes citées ici suggèrent que les agents anesthésiques pourraient être la cause d'une perte neuronale sélective chez nos patients. L'identification de ce phénomène chez des êtres humains s'avérerait très complexe, étant donné que la capacité de détecter une telle perte neuronale sélective pourrait se trouver au-delà de la sensibilité des techniques actuelles d'imagerie médicale. De plus, la capacité à définir la déficience fonctionnelle provenant de cette perte neuronale sélective est également difficilement mesurable. L'évaluation de cet effet pourrait être encore plus problématique chez une population pédiatrique, étant donné que le plein potentiel du système nerveux en développement et de l'individu pourrait prendre des années avec d'être accompli. Toutefois, si nous ne posons pas la question et ne testons pas l'hypothèse avec une méthodologie expérimentale reposant sur des bases solides, nous n'aurons pas de réponse.

L'enthousiasme qui pousse les chercheurs à développer des agents anesthésiques nouveaux et plus sécuritaires doit perdurer pour faire progresser la pratique de l'anesthésie. Un tel enthousiasme est évident dans le résumé d'une étude expérimentale qui déclare que le xénon a un "excellent profil d'innocuité " et est "très prometteur comme neuroprotecteur ». 6 Les déclarations de cette nature doivent être évaluées avec soin. Dans la littérature publiée, une recherche de "safety and anesthesia» a tiré 352 articles de ces vingt dernières années (Web of Science), alors qu'une recherche de "xenon and anesthesia » a retourné 244 références. Des recherches collectives pour « isoflura- ne, propofol, midazolam, ketamine and nitrous oxide and anesthesia » ont retourné plus de 20000 études. Néanmoins, nombre de ces agents ont le potentiel de provoquer la neuroapoptose, comme cela a été démontré dans des modèles animaux. De plus, il a été démontré que le xénon augmente l'incidence de nausées et de vomissements, et perturbe l'équilibre du flux sanguin cérébral vers les substances blanche et grise chez les humains. ${ }^{19,20}$ Il est évident que d'autres études sont nécessaires avant de pouvoir déterminer l'efficacité et l'innocuité d'une anesthésie au xénon. En outre, la découverte que des agents anesthésiques bien établis peuvent également induire une neuroapoptose soutient l'idée d'une surveillance post-commercialisation des médicaments afin de maintenir une vigilance continue en ce qui a trait au potentiel toxique d'un médicament.

Les efforts visant à se baser sur des approches scientifiques moléculaires perfectionnées pour concevoir des agents anesthésiques plus sécuritaires et plus efficaces sont démontrés dans les recherches en cours d'Orser, ${ }^{21}$ et d'autres, qui sont en train d'identifier des sous-types spécifiques du récepteur GABA qui pourraient constituer d'importantes 'cibles' pour les agents anesthésiques futurs. Des études cliniques et expérimentales continues, qui évalueraient l'effet des agents anesthésiques sur la santé et les lésions neuronales, offriront une meilleure compréhension des risques et bienfaits de l'anesthésie pour nos patients, et pourront peut-être mener au développement d'agents anesthésiques nouveaux et améliorés.

\section{References}

1 Cullen SC, Gross EG. The anesthetic properties of xenon in animals and human beings, with additional observations on krypton. Science 1951; 113: 580-2.

2 Franks NP, Dickinson R, de Sousa SL, Hall AC, Lieb $W R$. How does xenon produce anaesthesia? Nature 1998; 396: 324.

3 Rossaint R, Reyle-Hahn M, Schulte am Esch J, et al.; Xenon Study Group. Multicenter randomized comparison of the efficacy and safety of xenon and isoflurane in patients undergoing elective surgery. Anesthesiology 2003; 98: 6-13.

4 Goto T, Saito H, Shinkai M, Nakata $\Upsilon$, Ichinose F, Morita $S$. Xenon provides faster emergence from anesthesia than does nitrous oxide-sevoflurane or nitrous oxide-isoflurane. Anesthesiology 1997; 86: 1273-8.

5 Ma D, Hossain M, Pettet GK, et al. Xenon preconditioning reduces brain damage from neonatal asphyxia in rats. J Cereb Blood Flow Metab 2006; 26: 199-208. 
6 Hobbs C, Thoresen M, Tucker A, Aquilina K, Chakkarapani E, Dingley J. Xenon and hypothermia combine additively, offering long-term functional and histopathologic neuroprotection after neonatal hypoxia/ischemia. Stroke 2008; 39: 1307-13.

7 Cattano D, Williamson P, Fukui K, et al. Potential of xenon to induce or protect against neuroapotosis in the developing mouse brain. Can J Anesth 2008; 55: 429-36.

8 Jevtovic-Todorovic V, Hartman RE, Izumi $\Upsilon$, et al. Early exposure to common anesthetic agents causes widespread neurodegeneration in the developing rat brain and persistent learning deficits. J Neurosci 2003; 23: 876-82.

9 Olney JW, Young C, Wozniak DF, Jevtovic-Todorovic $V$, Ikonomidou C. Do pediatric drugs cause developing neurons to commit suicide? Trends Pharmacol Sci 2004; 25: 135-9.

10 Olney JW, Young C, Wozniak DF, Ikonomidou C, Jevtovic-Todorovic $V$. Anesthesia-induced developmental neuroapoptosis. Does it happen in humans? Anesthesiology 2004; 101: 273-5.

11 Todd MM. Anesthetic neurotoxicity: the collision between laboratory neuroscience and clinical medicine. Anesthesiology 2004; 101: 272-3.

12 Ikonomidou C, Bosch F, Miksa M, et al. Blockade of NMDA receptors and apoptotic neurodegeneration in the developing brain. Science 1999; 283: 70-4.

13 Johnson SA, Young C, Olney JW. Isoflurane-induced neuroapoptosis in the developing brain of nonhypoglycemic mice. J Neurosurg Anesthesiol 2008; 20: $21-8$.

14 Jevtovic-Todorovic V, Beals J, Benshoff N, Olney JW. Prolonged exposure to inhalational anesthetic nitrous oxide kills neurons in adult rat brain. Neuroscience 2003; 122: 609-16.

15 Lagasse RS. Anesthesia safety: model or myth? A review of the published literature and analysis of current original data. Anesthesiology 2002; 97: 1609-17.

16 Cheney FW, Posner KL, Lee LA, Caplan RA, Domino $K B$. Trends in anesthesia-related death and brain damage: a closed claims analysis. Anesthesiology 2006; 105: 1081-6.

17 Silverstein JH, Timberger M, Reich DL, Uysal S. Central nervous system dysfunction after noncardiac surgery and anesthesia in the elderly. Anesthesiology 2007; 106: 622-8.

18 Karkouti K, Djaiani G, Borger MA, et al. Low hematocrit during cardiopulmonary bypass is associated with increased risk of perioperative stroke in cardiac surgery. Ann Thorac Surg 2005; 80: 1381-7.

19 Coburn M, Kunitz O, Apfel CC, Hein M, Fries M, Rossaint $R$. Incidence of postoperative nausea and emetic episodes after xenon anaesthesia compared with propofol-based anaesthesia. Br J Anaesth 2008 [Epub ahead of print] PMID 1839792.

20 Rex S, Meyer PT, Baumert JH, et al. Positron emission tomography study of regional cerebral blood flow and flow-metabolism coupling during general anaesthesia with xenon in humans. Br J Anaesth 2008; 100: 667-75.

21 Bonin RP, Orser BA. GABA(A) receptor subtypes underlying general anesthesia. Pharmacol Biochem Behav 2008 [Epub ahead of print] PMID 18201756. 